\title{
PROPRIETA' DEI CENTRI CONJUGATI PRINCIPALI E DEI PIANI PRINCIPALI CONJUGATI, DEDOTTE DALLA CONSIDERAZIONE DEGLI ASSI DEI PENNELLI LUMINOSI, ED APPLICAZIONE DI ESSE AL CALCOLO DEGLI STROMENTI OTTICI COMPOSTI DI PIU' LENTI DELLE CUI GROSSEZZE SI DEBBA TENER CONTO. NOTA
}

DEL PROF. (). F. MOSSOTTI.

1.

Nell'estratto ragionato, che il Professore Cattaneo ba dato in questi Annali delle prime due Parti della mia Memoria sulla Teoria degli stromenti ottici, leggesi il seguente passo: "Queste applicazioni " cioe quelle falte alla spiegazione degli effetli ottici fra alcuni degli stromenti più in uso "lasciano però desiderarc che, come, sulle " prime, nella teoria delle lenti semplici aveva l'A. preso a ealcolo anche le gros" sezze delle medesime, cosi, anzi che trascurarle di poi, avesse cercato quale in" fluenza essa abbia, e ne'microscopi e nei cannocchiali e nel micrometro a doppia ” immagine. "In seguito il dotto Estensore ha indicato come l'impiego dei punti principali considerati da Gauss servirebbe all'oggetto, ed ha arricchito le sue osservazioni col notare delle nuove proprietá dei punti medesimi, e col far conoseere l'sistenza d'altre coppie di punti che godono di propricta analoghe.

Questo desiderio manifestato dal detto Professore, e quello nato in mie di dare miglior compimento alla mia Teoria, hanno richiamato la mia attenzione sui punti detti da Gauss principali, e mi sono provato a tentare, per mezzo della considerazione degli assi dei pennelli luminosi, una deduzione più semplice della loro esistenzi, ed un'interpretazione più esplicita della loro natura. I risultati delle ricerche fatte formeranno il soggetto d'un Appendice da pubblicarsi col seguito della Memoria, trattanto spero che non sarà discaro al lettore di questi Annali di conoscerne fin d'ora il contenuto.

2.

\section{Deduzione dei centri conjugati.}

La proprietà che serve a definire il raggio che costituisce l'asse d'un pentello luminoso che attraversa una lente è espressa $\left({ }^{*}\right)$, usando delle stesse denominazioni della Memoria che sono pure state conservate nell'Estratto di questi Annali, da

$$
\frac{\cos \mathrm{Y}_{2}}{v_{2}}=\frac{\cos \mathrm{Y}_{0}}{v_{0}}, \quad \text { e } \quad \frac{\cos Z_{2}}{v_{3}}=\frac{\cos Z_{0}}{v_{0}}:
$$

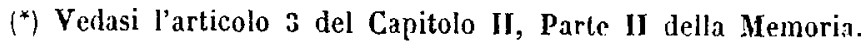

Tom. I. N N⿴. . 1858. 
cioè è quel raggio che entra ed esce dalla lente parallelamente a se stesso, se la lente è immersa in uno slesso mezzo, ovvero si rifrange come se passasse da uno ad un altro mezzo altraverso una superficie piana perpendicolare all'asse centrale, se la lente $\dot{c}$ interposta fra questi due mezzi.

Eliminando colle premesse equazioni $\cos \mathrm{Y}_{2}$ e $\cos \mathrm{Z}_{2}$ dalla seconda delle equazioni (8) e (9) date all'articolo 3 del Capitolo III, Parte I, $\left(^{*}\right)$ avremo per la determinazione dell'asse del pennello le seguenti

$$
0=\mathrm{P}_{3}^{(1)} y_{1}+\left(\mathbf{P}_{3}^{(2)}-1\right) \frac{\cos \mathrm{Y}_{\mathrm{o}}}{v_{0}}, \quad 0=\mathbf{P}_{3}^{(1)} z_{\mathrm{x}}+\left(\mathbf{P}_{3}^{(2)}-1\right) \frac{\cos Z_{0}}{v_{0}},
$$

ovvero, ponendo per $\cos \mathrm{Y}_{0}$ e $\cos \mathrm{Z}_{0}$ i loro valori, che, nella supposizione che il piano delle coordinate $x, y$ passi pel punto radiante, sono date da

$$
\cos Y_{o}=\frac{y_{1}-y_{0}}{\Delta_{0}}, \quad \cos Z_{o}=\frac{z_{1}}{\Delta_{0}},
$$

quest'altre due

$$
\left\{\mathrm{P}_{3}^{(1)}+\frac{1}{v_{0} \Delta_{\mathrm{o}}}\left(\mathrm{P}_{3}^{(2)}-1\right)\right\} y_{\mathrm{r}}=\frac{1}{v_{\mathrm{o}} \Delta_{\mathrm{o}}}\left(\mathrm{P}_{3}^{(2)}-1\right) y_{0}, \quad\left\{\mathrm{P}_{3}^{(1)}+\frac{1}{v_{0} \Delta_{\mathrm{o}}}\left(\mathrm{P}_{3}^{(2)}-1\right)\right\} z_{\mathrm{t}}=0 .
$$

La seconda di queste equazioni, eccettuando il caso che il valore di $\Delta_{0}$ annulli il coefficiente, esige che sia $z_{1}=0$; e ei mostra che l'asse del pennello sta nel piano passante per l'asse centrale, e pel punto raggiante: ponendo poi

$$
l_{0}=\frac{\mathbf{P}_{3}^{(2)}-1}{v_{0} \mathbf{P}_{3}^{(1)}}
$$

la prima prende la forma

$$
\left(1+\frac{l_{\mathrm{o}}}{\Delta_{\mathrm{o}}}\right) y_{\mathrm{t}}=\frac{l_{\mathrm{o}}}{\Delta_{\mathrm{o}}} y_{\mathrm{o}}
$$

dalla quale si ricava

$$
y_{\mathrm{r}}=\frac{l_{\mathrm{o}}}{\Delta_{\mathrm{o}}+l_{\mathrm{o}}} y_{\mathrm{o}} .
$$

Nella figura $1^{\text {a }}$. riportata in fine di questa Nota, simile a quella data nell'articolo 3 : del Capitolo II, Parte II, conduciamo dal punto raggiante $\mathbf{R}$ al punto $i$, pel quale

$\left({ }^{*}\right)$ Le equazioni (8) e (9) non sono citate sotto la stessa forma nell' Estratto, ma si ottengono direttamente ponendo nella seconda equazione della pagina 53 per le $u$ le loro espressioni date dalle (4) nella pagina precedente di esso, e sono

$$
\text { (a) } \quad y_{1}=\mathbf{P}_{2 i-2}^{\{1\}} y_{1}+\mathbf{P}_{2 i-2}^{(2)} \frac{\cos \mathbf{Y}_{0}}{v_{0}}, \quad \frac{\cos \mathbf{Y}_{i}}{v_{i}}=\mathbf{P}_{2 i-1}^{\{1\}} y_{\mathbf{1}}+\mathbf{P}_{2 i-1}^{\{2)} \frac{\cos \mathbf{Y}_{0}}{v_{0}} ;
$$

e due cquazioni simili si ottengono cambiando le $y$ in $z$, e le $\mathbf{Y}$ in $Z$. Abbiamo riferito queste equazioni perche avremo piu volte occasione di farne uso. 
l'asse del pennello penetra nella lente, la retta $\mathrm{R} i$, che prolungata incontri l' asse centrale IJ nel punto $K_{0}$. La similitudine dei triangoli $\mathrm{IK}_{0} \mathbf{R}, c_{0} \mathbf{K}_{0} i$ ci darà la proporzione

$$
\mathrm{IR}: c_{0} i=\mathrm{IK}_{\mathrm{o}}: c_{\mathrm{o}} \mathrm{K}_{\mathrm{o}},
$$

tal che essendo IR $=y_{0}, c_{0} i=y_{1}$, almeno nei limiti d'approssimazione entro cui sono rinchiuse le formole (2), ed $\mathbf{I K}_{0}=\Delta_{0}+c_{0} \mathbf{K}_{0}$, dal confronto del valore di $c_{0} K_{0}$, che si ricava da questa proporzione, con quello di $l_{0}$, che si deduce dalla precedente equazione, rileveremo dover essere $c_{0} \mathrm{~K}_{0}=l_{0}$.

Il raggio rappresentante l'asse del pennello che parte dal punto raggiante $R$ ha dunque la sua prima parte incidente così diretta, che taglia l'asse centrale in un punto $K_{o}$ distante dal centro di figura della superficie anteriore della lente di una quantità eguale ad $l_{0}$. Siccome questa quantità, dataci dalla formola (3) è soltanto funzione degli elementi fisici e geometrici della lente, e dei mezzi con cui è in contatto, ed è indipendente dalla posizione del punto raggiante $\mathrm{R}$, perciò conchiuderemo che qualunque sia la posizione di questo punto, quello d'intersezione della porzione incidente dell'asse del pennello luminoso coll' asse centrale della lente cadrà sempre nel luogo $\mathbf{K}_{o}$, vale a dire, le parti incidenti degli assi dei pennelli luminosi, che partono dai varii elementi dell' oggetto, prolungate, passeranno tulte per lo stesso punto $\mathbf{K}_{\mathrm{o}}$.

Consideriamo ora il corso retrogrado del raggio rappresentante la porzione convergente dell'asse del pennello luminoso, di modo che questo raggio parta dal fuoco $F$, coniugato di $R$, corrispondente alle coordinate $y, 0$ e $\Delta_{x}$ ed entri nella lente pel punto della superficie opposta $\mathrm{L}_{1} c_{1} \mathrm{~L}_{1}$, segnato $e$ nella figura, e le cui coordinate indicheremo con $y_{2}$ e $z_{2}$. Pel corso di questo raggio le equazioni corrispondenti alle (2) saranno giusta quanto fu detto all'articolo 6: del Capitolo IV, Parte I,

$$
0=-\mathbf{P}_{1}^{(2)} y_{2}+\left(\mathbf{P}_{1}^{(2)}-1\right) \frac{\cos \mathbf{Y}_{2}}{v_{2}}, \quad 0=-\mathbf{P}_{1}^{(3)} z_{2}+\left(\mathbf{P}_{1}^{(2)}-1\right) \frac{\cos Z_{2}}{v_{2}} .
$$

dalle quali eliminando $\cos \mathrm{Y}_{2}$ e $\cos \mathrm{Z}_{2}$ colle formole

$$
\cos \mathrm{Y}_{3}=\frac{y-y_{2}}{\Delta_{\mathrm{x}}}, \quad \cos \mathrm{Z}_{2}=-\frac{z_{2}}{\Delta_{\mathrm{I}}},
$$

ed invertendo gli indici superiori ed inferiori delle $P$, ciò che è lecito per quello chre fu detto nell'or eitato articolo, dedurremo $\left({ }^{*}\right)$

$$
\left\{\mathrm{P}_{3}^{(1)}+\frac{1}{v_{2} \Delta_{1}}\left(\mathrm{P}_{2}^{(1)}-1\right)\right\} y_{2}=\frac{1}{v_{2} \Delta_{1}}\left(\mathrm{P}_{2}^{(1)}-1\right) y, \quad\left\{\mathrm{P}_{3}^{(1)}+\frac{1}{v_{2} \Delta_{1}}\left(\mathrm{P}_{2}^{(1)}-1\right)\right\} z_{2:}=0
$$

(*) Vedi le equazioni (10) dell’'Estratto. 
equazioni le quali ci dicono che il raggio rappresentante l'asse della porzione cmergente del pennello incontra la seconda superficie della lente in un punto situato nel piano delle coordinate $x, y$, e che posto

$$
l_{1}=\frac{\mathrm{P}_{2}^{(1)}-1}{c_{2} \mathrm{P}_{3}^{(1)}}
$$

Lordinata $y_{2}$ del suo punto d'incontro colla detta superficie d̀ data dalla formola

$$
y_{2}=\frac{l_{1}}{\Delta_{1}+l_{1}} y \text {. }
$$

Questa formola $\dot{e}$ simile alla precedente (4), e confrontandola col valore di $c_{\mathbf{I}} \mathbf{K}_{\mathrm{r}}$, che si deduce dalla proporzione

$$
\mathrm{JE}: c_{\mathrm{I}} e=\mathrm{JK}_{\mathrm{I}}: c_{\mathrm{r}} \mathrm{K}_{\mathrm{I}},
$$

risultante dalla similitudine dei due triangoli $\mathbf{E K}_{\mathbf{I}} \mathbf{J} e \mathrm{~K}_{\mathrm{I}} c_{\mathrm{I}}$, che si ottengono col prolungare per indietro la porzione cmergente dell'asse del pennello sino ad incontrare l'asse centrale in $K_{1}$, ci mostra che $\dot{e} c_{\mathrm{I}} \mathrm{K}_{\mathrm{r}}=l_{\mathrm{r}}$, e che per essere $l_{\mathrm{r}}$ indipendente dalle quantila relative alla situazione del punto raggiante $R$, tutte le porzioni emergenti degli assi dei pennelli partiti dai diversi punti dell'oggetto concorrono virtualmente nel punto $\mathbf{K}_{\mathbf{r}}$.

Se col mezzo d'una lente sussidiaria si coneentrano nel punto $\mathbf{K}_{o}$ i raggi emanati da un punto luminoso situato sull' asse centrale della medesima, indi si colloca la lente che serve alle nostre considerazioni, Fig. I, in modo che il suo asse centrale coincida con quello della prima lente, e che il suo punto $K_{0}$ si sovraponga al punto $\mathbf{K}_{o}$ di riunione di detti raggi, si rileva da quanto è stato detto nell'articolo 3 : del Capitolo II, Parte II, sul centro ottico $\left(^{*}\right)$, e da quanto è stato testè dimostrato,

$\left({ }^{*}\right)$ La determinazione del centro ottico di una lente, che forma il soggetto del citato articolo, può conseguirsi, in un modo più conciso, e conforme alle considerazioni su cui s'aggira questa Nota, dalle equazioni (a) riferite a piè di pagina del numero 2 , omettendo quelle relative al piano delle $x, z$ che spariscono per essere $z_{4}$ e $\cos Z_{0}$ nulli. Quelle equazioni nel caso dell'asse d'un pennello pel quale deve sussistere la condizione (1), prendono la forma

$$
y_{2}=\mathbf{P}_{2}^{(1)} y_{1}+\mathbf{P}_{2}^{(2)} \frac{\cos \mathbf{Y}_{0}}{v_{0}}, \quad 0=\mathbf{P}_{3}^{(1)} y_{1}+\left(\mathbf{P}_{3}^{(2)}-1\right) \frac{\cos \mathbf{Y}_{0}}{v_{0}}
$$

Si elimini da queste $\cos \mathbf{Y}_{\mathrm{o}}$, e si riduca il coefficiente di $y_{\mathrm{c}}$ col mezzo della (3) del Capitolo IV, Parte I, e si otterrà

$$
y_{2}=-\frac{\mathbf{P}_{2}^{(1)}-1}{\mathbf{P}_{3}^{(2)}-1} y_{\mathrm{r}}=-\frac{v_{2} l_{\mathrm{r}}}{v_{0} l_{\mathrm{o}}} y_{\mathrm{I}}
$$

Se ora nell'equazione rappresentante il corso della porzione interna dell'asse del pennello, che $\grave{e}$

$$
y=\frac{y_{2}-y_{1}}{x_{2}-x_{1}} x+y_{1},
$$

poniamo $y=0$, per avere il valore $x^{\prime}$ dell'ascissa $x$ in cui esso interseca l'asse centrale, ed osserviamo 
che $i$ suddetti raggi, penetrati che siano nella lente in considerazione, anderanno tutti a passare pel centro ottico della medesima ed usciti dalla superficie opposta s'inclineranno in modo d'aver per fuoco virtuale il punto $\mathrm{K}_{\mathrm{I}}$, vale a dire, il centro ottico C sarà il fuoco conjugato del punto $\mathrm{K}_{0}$ rispelto alla superficie rifrangente $\mathrm{L}_{0} c_{0} \mathrm{~L}_{0}$, attraversata dai raggi incidenti, ed il punto $K_{x}$ il fuoco virtuale di $C$ rispetto alla superficie rifrangente $\mathrm{L}_{1} c_{\mathrm{r}} \mathrm{L}_{\mathrm{r}}$ attraversata dai raggi emergenti. Un effelto simile ma in ordine inverso si otterrebbe se $\mathrm{i}$ raggi emanati dal punto luminoso fossero diretti al punto $\mathrm{K}_{\mathrm{r}}$ per mezzo della lente sussidiaria interposta sul loro cammino, avanti di penetrare nella lente che consideriamo: l' immagine del detto punto sarebbe prima formata nel centro ottico $\mathrm{C}$, indi virtualmente nel fuoco coniugato $K_{o}$. Per evitare d'estendere al di là dei giusti confini queste analogic degli effelti prodotti dall'azione della lente sui raggi partiti da un punto luminoso, ed in seguito concentrati in $\mathbf{K}_{\text {o }}$ ovvero $\mathbf{K}_{\mathbf{r}}$, con quelli che la stessa azione produce sui raggi rappresentanti gli assi dei pennelli luminosi provenienti dai vari elementi d'un oggetto, giova tener presente che quantunque questi raggi siano riuniti realmente e poi virtualmente nei punti $\mathrm{C}$ e $\mathbf{K}_{\mathfrak{r}}$ ovvero $\mathbf{C}$ e $\mathbf{K}_{o}$ pure non produrrebbero come quelli emanati dal punto luminoso un'immagine, perchè $\mathrm{i}$ raggi costitucnti gli assi dei pennelli luminosi, avendo, ciascuno, un'origine diversa, come originati in punti diversi dell' oggetto, apparterrebbero a sistemi d'ondulazioni eterogenee che interferirebbero in gran parte fra loro, e non genererebbero tutl'al più che qualehe luce confusa (*).

I ragionamenti fatli fin qui suppongono, come viene rappresentato nella Fig. I"., che la lente sia convesso-convessa e sotlile, vale a dire, parlando analiticamente, che

che, $x_{1}$ ed $x_{2}$ dinotando le ascisse corrispondenti alle ordinate $y_{1}$ ed $y_{2}$, si può prendere, nel grado d'approssimazione in eui stiamo, $x_{2}-x_{1}=h_{2}$, troveremo

$$
x^{\prime}=\frac{l_{\mathrm{L}}}{l_{\mathrm{L}}+i_{0}} h_{3} .
$$

Questo valore di $x^{\prime}$, essendo indipendente dalle coordinate $\Delta_{0}$ ed $y_{0}$ del punto raggiante, ci prova che, se la lente è investita da pia pennelli procedenti da punti luminosi diversi, tutti gli assi di questi pennelli intersecano l'asse centrale in uno stesso punto che è il centro ottico della lente.

Il valore di $x^{\prime}$ ci dà l'ascissa del centro ottico preso dal centro di figura della superficie anteriore della lente. Volendo avere l'ascissa $x^{\prime \prime}$ presa dal centro di figura della superticie posteriore si osserveri che è $x^{\prime \prime}=x^{\prime}-h_{2}$, c quindi si troverà

$$
x^{\prime \prime}=-\frac{l_{0}}{l_{1}+l_{0}} h_{2} .
$$

(*) Il considerare separatamente i raggi emanati da ciascun elunento dell' oggetto come componenti. dei pennelli distinti, ed esaminare il corso dei loro assi successivi è cosa assai importante nelle teorie dellottica, come lo comprova la semplicità con cui abbiamo dedntto le proprietà degli stromenti ottici dalle equazioni segnate (6) alla pagina 53 dell'Estratto, nelle quali non appariscono come variabili le coordinate del punto d'emanazione dei raggi e quelle del punto d'incidenza. I primi scrittori d'ottica che impiegavano invece le coordinate di quest'ultimo punto e le direzioni dei raggi sono caduti talvolta in dimostrazioni confuse, e deduzioni erronee. Vedasi ancbe la nota dell'articolo 7 , Capitolo II, Parte II della Memoria. 
si abbia

$$
p_{1}<0, \quad p_{3}<0, \quad \mathrm{P}_{3}^{(1)}<0
$$

ossia

$$
h_{2}<-\frac{1}{v_{\mathrm{I}}}\left(\frac{1}{p_{3}}+\frac{1}{p_{\mathrm{I}}}\right)
$$

nel qual caso le distanze $l_{0}$ ed $l_{\mathbf{1}}$ ottengono valori positivi, ed i punti $\mathrm{K}_{\mathrm{o}}$ e $\mathbf{K}_{\mathbf{1}}$ sono situati nell'interno della lente. Le posizioni dei punti $K_{0}$ e $K_{\mathbf{r}}$ risulterebbero però diverse a seconda che una o due delle tre relazioni $(r)$ non fossero soddisfatte. Senza discutere i vari casi che si possono presentare, ciò che ci devierebbe troppo dal nostro scopo, sceglieremo per esempio quello in cui le quantità $p_{\mathrm{r}}$ e $p_{3}$ rimanendo negative, la funzione $\mathrm{P}_{3}^{(1)}$ sia di valore posilivo, cioè che la lente sia sufficientemente grossa da avere

$$
h_{2}>-\frac{1}{v_{\mathrm{I}}}\left(\frac{1}{p_{3}}+\frac{1}{p_{\mathrm{I}}}\right) .
$$

In questo caso $\mathrm{i}$ valori di $l_{0}$ ed $l_{\mathbf{x}}$ risulteranno negativi, ed $\mathrm{i}$ punti $\mathbf{K}_{\mathrm{o}}$ e $\mathbf{K}_{\mathbf{x}}$ saranno situatial di fuori della lente, come nella Figura II'., nella quale le linee corelative sono marcate colle stesse letterc che nella Figura $I^{\prime}$.

Dalla detta Figura $I^{a}$. si rileva che se esistesse in uno dei due punti $\mathbf{K}_{0}$ e $\mathbf{K}_{\mathbf{I}}$ un elemento luminoso per se stesso si produrrebbe un'immagine reale del medesimo nell'altro punto.

Da quanto abbiamo esposto chiaro apparisce che $i$ punti $K_{o} C_{1} K_{1}$ oltre alla particolarita di poter divenire, secondo $\mathrm{i}$ casi, fuochi conjugati reali o virtuali d'un elemento luminoso situato in uno di essi, o d'un'immagine dello stesso clemento penetrata nella lente dal di fuori, godono la proprietà caratteristica d'essere, in ogni caso, i punti di reale o virtuale concorso delle tre parti degli assi dei pennelli, che, partili dai vari elementi d'un oggretto posto ad una lontananza qualunque, ma ad una distanza dall'asse comparativamente molto più piccola, investono una lente. Il punto $\mathbf{K}_{0}$ è il luogo di concorso delle parti incidenti degli assi dei pennelli, il punto $\mathrm{C}$ quello delle parti interne, ed il punto $\mathbf{K}_{\mathbf{I}}$ quello delle parti emergenti: per tale motivo dando a questi punti il nome di centri, conserveremo al punto $\mathrm{C}$ il nome comunemente usato di centro ottico, ed indicheremo $i$ punti $K_{0}$ e $K_{r}$ con quello di centri conjugati principali $\left({ }^{*}\right)$.

$\left(^{*}\right)$ Questa denominazione ci sembra più espressiva di quella di punti principali introdotta da Gauss. la quale non enuncia alcuna proprietà dei punti suddetti.

A questo proposito osserveremo che la proprietà d'intersecarsi, realmente o virtualmente sull'asse centrale in tre punti consecutivi, è comune, ciò che forse non è stato ancora avvertito, a tutti i sistemi di raggi pei quali $\cos Y_{2}: \cos Y_{0}$ e $\cos Z_{2}: \cos Z_{0}$ hanno un rapporto eguale e costante; e che al sistema, in cui questo rapporto eguaglia $v_{2}$ : $v_{0}$ va unita un'altra proprietà caratteristica, ed è che $\mathrm{i}$ piani perpendica- 
3.

\section{Piani principali conjugati.}

Un'altra proprietà notevole dei centri conjugati si è, che, condotti per essi due piani $p_{0} \mathrm{~K}_{\mathrm{o}} p_{\mathrm{o}}, p_{\mathrm{I}} \mathrm{K}_{\mathrm{r}} p_{\mathrm{I}}$, Figura $\mathrm{I}^{\mathrm{a}}$. e $\mathrm{II}^{\mathrm{a}}$., perpendicolarmente all'asse centrale, la porzione incidente $R \mu$ d'un raggio qualunque, e quella emergente $\mu_{\mathbf{x}} \mathbf{E}$ dello stesso raggio incontrano respettivamente questi piani in due punti situati sopra una retta parallela all'asse centrale.

In fatti le due equazioni della porzione incidente del raggio passante pel punto d'incidenza, essendo, nei limiti adottati d'approssimazione, in eui si può prendere $\cos \mathbf{X}_{0}=1$, date da

$$
y-y_{\mathrm{r}}=\left(x-x_{\mathrm{i}}\right) \cos \mathrm{Y}_{0}, \quad z-z_{\mathrm{x}}=\left(x-x_{\mathrm{1}}\right) \cos \mathrm{Z}_{\mathrm{o}} ;
$$

se facciamo in esse $x-x^{\prime}=l_{0}$, per ottenere le coordinate del punto d'incontro della detta porzione col primo piano $p_{0} \mathrm{~K}_{\mathrm{o}} p_{0}$, e poniamo per $\cos \mathrm{Y}_{\mathrm{o}}, \cos \mathrm{Z}_{\mathrm{o}}$ i loro valori più volte citati, avremo per le coordinate $y^{\prime}$ e $z^{\prime}$ di questo punto

$$
y^{\prime}=y_{\mathrm{x}}+\frac{l_{\mathrm{o}}}{\Delta_{\mathrm{o}}}\left(y_{\mathrm{x}}-y_{\mathrm{o}}\right) ; \quad z^{\prime}=z_{\mathrm{x}}+\frac{l_{\mathrm{o}}}{\Delta_{\mathrm{o}}}\left(z_{\mathrm{x}}-z_{\mathrm{o}}\right) .
$$

Ora le due equazioni del raggio emergente rappresentate in generale da quelle segnate (8) nell'Estratto, ci danno, quando si la $n=2$, e si pone $x-\mathbf{H}_{2}=-l_{\mathrm{t}}$, per dedurre $i$ valori delle coordinate $y^{\prime \prime}$ e $z^{\prime \prime}$ del punto d'incontro della porzione del raggio emergente, $e E$, del raggio del secondo piano $p_{\mathbf{I}} \mathbf{K}_{1} p_{\mathbf{I}}$

$$
\begin{aligned}
& y^{\prime \prime}=\left(Q_{2}^{(1)}-v_{2} l_{1} Q_{8}^{(1)}\right) y_{1}-\frac{1}{v_{0} \Delta_{0}}\left(\mathbf{P}_{2}^{(2)}-v_{2} l_{1} \mathbf{P}_{3}^{(2)}\right) y_{0} \\
& z^{\prime \prime}=\left(Q_{2}^{(1)}-v_{2} l_{1} Q_{3}^{(1)}\right) z_{1}-\frac{1}{v_{0} \Delta_{0}}\left(\mathbf{P}_{2}^{(2)}-v_{2} l_{1} P_{3}^{(2)}\right) z_{0}
\end{aligned}
$$

le quali, ridotte colla sostituzione dei rispettivi valori di $Q_{2}^{(1)}$ e $Q_{3}^{(1)}$, somministratici dalle (5) dell'Estratto, e col mezzo della prima delle (7), prendono pure la forma

$$
y^{\prime \prime}=y_{\mathrm{x}}+\frac{l_{\mathrm{o}}}{\Delta_{0}}\left(y_{\mathrm{x}}-y_{0}\right), \quad z^{\prime \prime}=z_{\mathrm{x}}+\frac{l_{\mathrm{o}}}{\Delta_{\mathrm{o}}}\left(z_{\mathrm{I}}-z_{0}\right),
$$

lari all'asse centrale e passante pel primo ed ultimo dei tre punti d'intersezione tagliano un raggio qualunque d'un pennello in due punti situati sopra una stessa parallela all' asse centrale; proprietà notevole scoperta dal Gauss, che dimostreremo nell'articolo seguente, e che è tanto utile nelle applicazioni. Il nome di centri conjugati, dato dal Sig. Biot, ai due punti d' intersezione coll'asse delle porzioni incidenti ed emergenti dei raggi del sistema, in cui il detto rapporto è eguale all'unità, non appartiene quindi esclusivamente ad essi ma è applicabile ai punti analoghi di tutti i sopra indicati sistemi di raggi, e l' epiteto principali serve a distinguere $\mathbf{i}$ due punti spettanti al sistema considerato da Grauss, che godono di naggiori e più utili proprietà. 
dunque arremo

$$
y^{\prime \prime}=y^{\prime}, \quad z^{\prime \prime}=z^{\prime} ;
$$

mostrandoci cosi che $\mathrm{i}$ due punti determinati appartengono ad una retta parallela all' asse delle $x$, ossia all'asse centrale.

Nelle Figure $I^{2}$. e $I^{2}$. le rette $m m_{\mathrm{I}}$ ed $n n_{\mathrm{I}}$ marcano respettivamente le parallele su cui stanno le intersezioni dei raggi $\mathrm{R} \mu \mu_{\mathbf{x}} \mathrm{E}, \mathbf{R} \nu_{\mathbf{x}} \mathrm{E}$ laterali all'asse del pennello RiCeE.

Ai due detti piani condotti perpendicolamente all' asse centrale pei due centri conjugati principali, e che sono rispetlivamente intersecati da un raggio qualunque del pennello luminoso in punti egualmente situati su di essi daremo, ad imitazione del Causs, il nome di piani conjugati principali, o semplicemente di piani principali.

4.

Determinazione della lunghezza focale di una lente fittizia, da sostituirsi alla reale, mediante la quale si riduce più semplice il calcolo degli effetti d'uno stromento ottico.

Se s' immagina che spariscano tulte le parti della Figura $\mathbf{I}^{a}$. o $\mathbf{l I}^{\mathrm{a}}$. spetlanti alla forma della lente, e che $\mathrm{i}$ due piani centrali coniugati $p_{0} \mathbf{K}_{\mathrm{o}} p_{\mathrm{o}}, p_{\mathrm{r}} \mathbf{K}_{\mathrm{r}} p_{\mathbf{r}}$ vadano a riunirsi col piano $c \mathrm{C} c$ condotto pel centro oltico perpendicolarmente all'asse centrale, e che questo piano rimanendo parallelo a se stesso passi alla distanza del punto raggriante $\mathbf{R}$ a cui sta il piano $p_{0} \mathbf{K}_{\mathrm{o}} p_{0}$ nel caso reale, e si suppone inoltre che, nel luogo ove $\dot{\mathrm{c}}$ venuto a collocarsi il piano $p_{0} \mathrm{C} p_{0}$, esista una lente di grossezza trascurabile, dotata d'una lunghezza focale $\varphi$, che soldisfaccia all'erquazione

$$
\frac{1}{v_{2}\left(\Delta_{\mathrm{I}}+l_{\mathrm{I}}\right)}=\frac{1}{v_{2} \varphi}-\frac{1}{v_{0}\left(\Delta_{0}+l_{\mathrm{o}}\right)},
$$

simile a quella segnata ( 7$)^{\prime \prime}$ nell'articolo 2 del Capitolo II, Parte II, che vale rigorosamente per una lente infinitamente sottile $\left({ }^{*}\right)$ ̀̀ chiaro, che in tale ipotesi l'asse del pennello luminoso cntrerà ed uscirà da questa lente percorrendo una linea rella, se la lente è immersa in uno slesso mezzo omogenco, ovvero, spezzata secondo la legge data dall' equazione (1), se il mezzo anteriore e quello posteriorc alla lente sono differentemente rifrangenti, e che tanto le dirczioni di tulli i raggi del pennello luminoso, quanto le posizioni dei punti in cui essi sono intersecati dai piani centrali rimaranno eguali come nel caso della lente reale; solo la distanza reciproca dei due fuochi coniugati $\mathbf{R}$ ed $\mathbf{E}$ sarà diminuita dell' intervallo $\mathbf{K}_{\mathbf{o}} \mathbf{K}_{\mathbf{I}}$. Da

( ) li equazione citata suppone che la lente sia immersa in uno stesso mezzo, per cui si abbia $c^{\prime}=r_{0}$ : se la lente separasse due mezi differenti, i facile di vedere che prenderebhe la forma

$$
\frac{1}{r_{2} d_{1}}=\frac{1}{v_{2} F}-\frac{1}{v_{0} \Delta_{0}}
$$


ciò ne segue, che se calcoleremo la distanza conjugata $\Delta_{1}+I_{1}$, e le coordinate del fuoco coniugato corrispondente alla lente fittizia colle formole $(7)^{\prime \prime}$ ed $(8)^{\prime \prime}$, date nel luogo sopra citato, impiegando in esse la distanza focale $\varphi$ in luogo di $F$, e la distanza conjugata $\Delta_{0}+l_{0}$ del punto raggiante in luogo di $\Delta_{0}$, indi partendo dal secondo piano principale applichiamo all'asse centrale un aseissa eguale al valore calcolato di $\Delta_{x}+l_{x}$, ed all'estremità di quest'ascissa due coordinate eguali agli ottenuti valori di $y \mathrm{e} z$, avremo segnato la posizione del fuoco conjugato spettante alla lente reale. Per eseguire questo calcolo non ci rimarrà altro a conoscere se non se il valore della lunghezza focale $\varphi$, da cui l'equazione ( 7 ) sia soddisfatta.

Per procacciarci questo valore assumiamo l'equazione (10) dell'Estratto, in cui siasi fatto $n=2$, e siansi poste per le $Q$ le loro espressioni segnate (5), pure nell'Estratto, e, per introdurre le distanze focali conjugate $\Delta_{0}+l_{0}$, e $\Delta_{\mathrm{x}}+l_{\mathrm{s}}$, seriviamo in essa $\Delta_{0}+l_{0}-l_{0}$ in loogo di $\Delta_{0}$, e $\Delta_{1}+l_{\mathrm{r}}-l_{1}$ in luogo di $\Delta_{1}$; la citata equazione ei darà

$$
\Delta_{1}+l_{1}=-\frac{1}{v_{2}} \frac{v_{0}\left(\Delta_{0}+l_{0}\right) \mathbf{P}_{2}^{(1)}+\mathbf{P}_{2}^{(3)}-v_{0} l_{0} \mathbf{P}_{2}^{(1)}}{v_{2}\left(\Delta_{0}+l_{0}\right) \mathbf{P}_{3}^{(1)}+\mathbf{P}_{3}^{(2)}-v_{0} l_{0} \mathbf{P}_{3}^{(1)}}+l_{\mathrm{r}},
$$

e riducendo il secondo membro alla stessa denominazione, ed osservando che, coll: sostituzione dei rispettivi valori di $l_{\mathrm{o}}$ ed $l_{\mathrm{1}}$ datici dalle (3) e (5), e colle riduzioni somministrateci dalla prima delle (7) (Est.). la quantitia

$$
v_{2} l_{1} \mathbf{P}_{3}^{(2)}-\mathbf{P}_{2}^{(2)}-v_{0} l_{0}\left(v_{2} l_{1} \mathbf{P}_{3}^{(1)}-\mathbf{P}_{2}^{(1)}\right)
$$

risulta identicamente nulla, si troverà

$$
\Delta_{\mathrm{x}}+l_{\mathrm{x}}=-\frac{v_{0}\left(\mathbf{P}_{2}^{(1)}-v_{2} l_{\mathrm{x}} \mathbf{P}_{3}^{(1)}\right)\left(\Delta_{0}+l_{0}\right)}{v_{2} v_{0}\left(\Delta_{0}+l_{0}\right) \mathbf{P}_{3}^{(1)}+v_{2}\left(\mathbf{P}_{3}^{(2)}-v_{0} l_{0} \mathbf{P}_{3}^{(1)}\right)},
$$

ovvero rovesciando un membro e l'altro

$$
\frac{1}{\Delta_{\mathrm{I}}+l_{1}}=-\frac{v_{2} \mathrm{P}_{3}^{(1)}}{\mathbf{P}_{2}^{(1)}-v_{2} l_{1} \mathbf{P}_{3}^{(1)}}-\frac{v_{2}}{v_{0}} \frac{\mathbf{P}_{8}^{(2)}-v_{0} l_{0} \mathbf{P}_{8}^{(1)}}{\mathbf{P}_{2}^{(1)}-v_{2} l_{1} \mathbf{P}_{2}^{(1)}} \cdot \frac{1}{\Delta_{0}+l_{0}} .
$$

Ora si può osservare che la quantità $\mathbf{P}_{2}^{(1)}-v_{2} l_{\mathbf{x}} \mathbf{P}_{3}^{(1)}$, e $\mathbf{P}_{3}^{(2)}-v_{0} l_{0} \mathbf{P}_{3}^{(1)}$ sono suscettibili d'essere trasformate in due modi. Primieramente mettendo per $l_{o}$ ed $l_{1}$ i valori. e secondariamente facendo uso delle espressioni delle lunghezze focali

$$
F_{2}^{(1)}=-\frac{\mathbf{P}_{2}^{(1)}}{v_{3} \mathbf{P}^{1}} \quad, \quad F_{1}^{(2)}=-\frac{\mathbf{P}_{3}^{(2)}}{v_{0} \mathbf{P}_{3}^{(1)}},
$$

che possono dedursi dalle (22) dell'Estratto.

Per mezzo della duplice sostituzione orat accennata si trova

$$
\mathbf{P}_{a}^{(1)}-v_{2} l_{1} \mathbf{P}_{3}^{(1)}=1=-v_{2} \mathbf{P}_{3}^{(1)}\left(\mathbf{F}_{2}^{(1)}+l_{\mathbf{1}}\right), \quad \mathbf{P}_{3}^{(2)}-v_{0} l_{0} \mathbf{P}_{3}^{(1)}=\mathbf{1}=-v_{0} \mathbf{P}_{3}^{(1)}\left(\mathbf{F}_{1}^{(2)}+l_{0}\right) .
$$

Toin. 1. N. $N^{\circ} .5 .1858$. 
donde ricavasi

$$
v_{2}\left(\mathbf{F}_{2}^{(1)}+l_{1}\right)=v_{0}\left(\mathbf{F}_{1}^{(2)}+l_{0}\right)=-\frac{1}{\mathbf{P}_{3}^{(1)}}
$$

La precedente equazione (8) diverrà quindi coll'uso di questi valori

$$
\frac{1}{v_{2}\left(\Delta_{1}+l_{1}\right)}=-\mathbf{P}_{3}^{(1)}-\frac{1}{v_{0}\left(\Delta_{0}+l_{0}\right)},
$$

lit quale confrontata colla supposta segnata (7), ci mostra che la dimandata lunghezza focale 0 , ̀̀ data da

$$
\varphi=-\frac{1}{v_{2} \mathbf{P}_{3}^{(1)}}
$$

c perció, che, in virtù della (8), l'equazione (10) si può anche metlere solto una delle due forme

$$
\frac{1}{v_{z}\left(\Delta_{1}+l_{1}\right)}+\frac{1}{v_{0}\left(\Delta_{0}+l_{0}\right)}=\frac{1}{v_{2}\left(\mathrm{~F}_{2}^{(1)}+l_{\mathrm{r}}\right)}=\frac{1}{v_{0}\left(\mathrm{~F}_{1}^{(2)}+l_{0}\right)} .
$$

Nel dedurre l'esistenza e le proprictà dei centri conjugati principali dei piani principali e della lunghezza focale della Iente filtizia non abbiamo preso in considerazione che una sola lente, perchè è principalmente impiegando le proprietà dei delti elementi relativi a ciascuna lente che il calcolo degli stromenti ottici può essere abbreviato, quando si voglia tener conto della grossezza delle lenti. Non omctteremo però di osservare che basta di cambiare all'indice delle $\mathrm{P}$ il valore 2 , che gli è stato dato, nell'indice generale $n$, che tutte le formole precedentemente trovate, e quelle che hanno servito alle loro riduzioni sussisteranno ancora, e saranno applicabili alla determinazione dei delli elementi per un sistema composto di un numero qualunque di lenti. Vedasi anche l'articolo 1 del Capitolo III, Parte II.

5.

Calcolo degli effetti d'uno stromento ottico coll'impiego delle distanze dei centri conjugati principali, e delle relative lunghezze focali delle lenti fittizie.

Il vantaggio che porge la considerazione dei centri coniugati principali, e della lunghezza focale della lente fittizia, consiste principalmente nel fornirci il mezzo di tener conto della grossezza delle lenti nel calcolo del corso di un raggio luminoso qualunque in un'istromento ottico calcolando prima il corso dello stesso raggio come se le dette grossezze fossero trascurabili, c poi surrogando alle lunghezze focali delle lenti ed alle loro distanze reciproche quelle che competono alle lenti filtizie date ne precedenti articoli. Per tal modo si viene ad abbassare, come abbiamo visto nell'ar- 
ticolo 6 del Capitolo II, Parte II, della metà l'indice delle funzioni P, il cui numero di termini va elevandosi così rapidamente col erescere del loro indice da renderne presto il calcolo impraticabile, e si sostituisce un processo comparitivamente mollo più semplice.

Per vedere come ciò succeda, noteremo primieramente che basta gettar l'occhio sulla seconda parte della Figura III $^{2}$., nella quale si suppone che $\mathrm{i}$ piani principali di ciascuna lente dello stromento siano compenetrati con quelli passanti pel centro ottico rispettivo, e che l'intiero sistema sia traslocato in modo che il primo dei delti piani verso l'oggetto si trovi alla medesima distanza da questo, a cui nel sistema reale sta il piano principale anteriore della prima lente, e confrontare questa seconda parte colla prima rappresentante lo stromento per iscorgere che le direzioni, che prendr. successivamente un raggio qualunque d'un pennello luminoso, ed i punti in cui questo raggio sárà intersecato dai piani principali riuniti per coppie, saranno i medesimi di quando operano le grossezze delle lenti. Se quindi, impiegando $i$ dati appartenenti alle lenti fittizie, determineremo colle formole (5) e (6) del Capitolo II, Parte II il valore delle coordinate del fuoco coniugato dell'intero sistema, questi valori coincideranno con quelli che si otterrebbero dalle stesse equazioni per le coordinate del fuoco del sistema reale, prese partendo dal piano principale conjugato posteriore dell'ultima lente, nel caso che s'abbia riguardo alla grossezza delle lenti.

Per eseguire il calcolo delle coordinate del fuoco conjugato nel sistema delle lenti fittizie, per mezzo delle equazioni (5) e (6), bisogna conoscere quali valori devono sostituirsi in luogo delle coordinate $\Delta_{0}, y_{0}, z_{0}$ del punto radiante, ed in luogo degli elimenti $p_{\mathrm{I}}, p_{2}, p_{3}$, e componenti le funzioni $Q$, al quale oggetto serviranno le seguenti rimarche.

1. Le coordinate $y_{0}$ e $z_{0}$ rimangono eguali a quelle del sistema reale, la sola distanza $\Delta_{o}$ deve essere cambiata in $\Delta_{o}+l_{0}$ pel sistema fillizio.

2. Le $p$ con indice pari multiplo dispari di 2 , contenute nella serie

$$
p_{2}, p_{6}, p_{\mathrm{ro}} \ldots p_{h i-3},
$$

essendo, giusta le formole $(6)^{\prime}$ del Capitolo III, Parte I, proporzionali alle grossezze delle lenti, che sono nulle nel caso delle lenti fittizie, le dette $p$ saranno pure tutte nulle.

3. Le $p$ con un indice multiplo di $h$ sono date, giusta le citate formole, dal prodotto della velocità della luce nel mezzo che separa due lenti successive multiplicata per la loro distanza reciproca. Se indichiamo con $\Delta_{1}, \Delta_{2}, \Delta_{3} \ldots \Delta_{2 n}$ Ie distanze dei fuochi conjugati dai centri di figura delle rispettive superficie delle lenti nel sistema reale, e con $l_{\mathrm{o}}, l_{\mathrm{r}}, l_{2} \ldots l_{n}$ quelle dei corrispondenti piani principali, le prime essendo prese verso l'esterno della respettiva superficie della lente quantio 
i loro valori sono positivi, e verso l'interno quando sono negativi, e le seconde viceversa; è facile di vedere che le distanze reciproche dei piani saranno date da

$$
\Delta_{1}+l_{1}+\Delta_{2}+l_{2}, \Delta_{3}+l_{3}+\Delta_{4}+l_{4} \ldots \Delta_{2 n-1}+l_{2 n-1}+\Delta_{2 n}+l_{2 n}:
$$

ma nel caso reale si ha

$$
\Delta_{1}+\Delta_{2}=h_{3}, \quad \Delta_{3}+\Delta_{4}=h_{5} \ldots \Delta_{2 n-1}+\Delta_{2 n}=h_{2 n+1}
$$

quindi introducendo questi valori, quelli delle $p$ con indice multipli di $h$ componenti la serie

$$
p_{4}, \quad p_{8}, \ldots \ldots p_{2 n}
$$

saranno dati da

$$
v_{2}\left(h_{3}+l_{1}+l_{2}\right), \quad v_{4}\left(h_{5}+l_{3}+l_{4}\right) \ldots v_{2 n}\left(h_{2 n+1}+l_{2 n-1}+l_{2 n}\right) .
$$

In quanto alle $p$ con indice dispari, queste secondo le osservazioni fatte nell'articolo 6 del Capitolo II, Parte II, non entreranno nelle nostre formole che sommate per coppie, cioè non avremo a considerare che i termini delle serie

$$
p_{\mathrm{r}}+p_{3}, \quad p_{5}+p_{7} \ldots p_{2 n-\mathrm{I}}+p_{2 n+\mathrm{r}}
$$

che giusta l'articolo testè citato eguagliano ciascuno il respetlivo valore inverso del prodotto della velocità della luce nel mezzo in cui passa il raggio uscendo da una lente per la lunghezza focale della medesima, ossiano rappresentano $i$ successivi valori di

$$
\frac{1}{v_{2} \varphi_{1}}, \quad-\frac{1}{v_{4} \varphi_{2}} \cdots \cdots \frac{1}{v_{2 n} \varphi_{n}},
$$

the come abbiamo visto nell'articolo precedente sono espressi da

$$
\frac{1}{v_{2}\left(f_{1}+l_{\mathrm{x}}\right)}, \quad \frac{1}{v_{4}\left(f_{2}+l_{3}\right)} \cdots \cdots \frac{1}{v_{2 n}\left(f_{n}+l_{2 n-1}\right)} .
$$

Ma se noi supponiamo di fare colle citate formole (5) e (6), il calcolo approssimativo del fuoco conjugato del sistema reale, traseurando le grossezze delle lenti, dobbiamo, come è stato notato nell'articolo 6 testè menzionalo, fare le $p$ della serie (13) tutte nulle, sostituire alle $p$ comprese nella serie (14) respettivamente le quantità

$$
v_{2} h_{3}, \quad v_{4} h_{5} \ldots v_{2 n} h_{2 n+\mathrm{I}}
$$

ed alle coppie delle $p$ con indici dispari, componenti la serie (16), le quantità

$$
\frac{1}{v_{2} f_{\mathrm{x}}}, \frac{1}{v_{4} f_{2}} \cdots \cdots \frac{1}{v_{2 n} f_{n}}
$$

dunque se nelle formole ottenute col calcolo approssimativo, in cui siano trascurate le grossezze delle lenti, cambiamo le quantità della serie $(14)^{\prime}$ in quelle della serie 
PURA ED APPLICATA.

(14), e le quantità della serie $(17)^{\prime}$ in quelle della (17), otterremo dalle medesime formole quelle corrispondenti al sistema fittizio, applicabili al caso in cui si voglia tener conto della grossezza delle lenti.

Facendo pertanto le indicate sostituzioni nelle formole, ritrovate negli articoli 4 , 7 ed 8 del Capitolo II Parte II, spettanti al microscopio semplice, ai telescopi di Kepler e Galileo, ed al dinametro e prendendo per $\Delta_{0}$ la distanza dell oggetto dal primo piano principale del sistema, espresso da $\Delta_{0}+l_{0}$, e per $\Delta$ la distanza dell'immagine presa dall'ultimo piano principale, espressa da $\Delta+l$, si otterranno le rispettive formole, nel caso che le grossezze delle lenti non debbano essere trascurate.

Noteremo che i valori di $l_{0}$ ed $l_{\mathrm{x}}$ espressi per mezzo dei raggi di curvatura della lente e della sua grossezza, non che delle velocita della luce nel mezzo che la compone, e di quelli che le sono in contatto, si ottengono ponendo nelle formole (3) e (5)

e poi prendendo

$$
1-\frac{v_{\mathrm{x}}}{v_{\mathrm{o}}}=u_{\mathrm{r}}, \quad 1-\frac{v_{\mathrm{x}}}{v_{\mathrm{z}}}=u_{2}
$$

$$
l_{0}=\frac{\left(1-u_{\mathrm{x}}\right) u_{\mathrm{x}} \rho_{\mathrm{I}} h_{2}}{u_{\mathrm{x}} \rho_{\mathrm{I}}-u_{\mathrm{2}} \rho_{2}-u_{\mathrm{x}} u_{2} h_{2}}, \quad l_{\mathrm{x}}=-\frac{\left(1-u_{2}\right) u_{2} \rho_{2} h_{2}}{u_{\mathrm{x}} \rho_{\mathrm{I}}-u_{2} \rho_{2}-u_{\mathrm{x}} u_{2} h_{2}} .
$$

Quando la lente è immersa nello stesso mezzo si ha $v_{0}=v_{2}$ ed $u_{\mathrm{r}}=u_{2}$, e quindi le due formole precedenti danno

$$
l_{\mathrm{o}}=\frac{\left(1-u_{\mathrm{r}}\right) \rho_{\mathrm{I}} h_{2}}{\rho_{\mathrm{r}}-\rho_{2}-u_{\mathrm{x}} h_{2}}, \quad l_{\mathrm{I}}=-\frac{\left(1-u_{\mathrm{I}}\right) \rho_{2}}{\rho_{\mathrm{I}}-\rho_{2}-u_{\mathrm{x}}} \frac{h_{\mathrm{x}}}{h_{2}} .
$$

Terminerò questa Nota col ringraziare il Prof. Cattaneo d'avermi offerto l'occasione di supplire ad un'omissione nella mia Teoria degli stromenti ottici, ciò che parmi di aver conseguito non senza qualche novità. 